\title{
Direct oral anticoagulants: a treatment option in oncological patients
}

\author{
Johannes B. Jaeger · Thomas Jaeger · Clemens Feistritzer $(D$
}

Received: 19 January 2019 / Accepted: 5 March 2019 / Published online: 20 March 2019

(C) The Author(s) 2019

\begin{abstract}
Summary Venous thrombosis and pulmonary embolism are common complications in patients with active cancer. For many years low molecular weight heparins (LMWH) have been the preferred treatment option for cancer patients, because of their superiority over vitamin $\mathrm{K}$ antagonists, which bear high risk of interaction and unsatisfactory anticoagulation. With the introduction of direct oral anticoagulants (DOAC) a second oral treatment option appeared. However, recommendations for the use of DOACs in the treatment of cancer-associated venous thromboembolic events were only given recently. DOAC possess a different side effect profile regarding drug/drug interactions. Recent publications revealed a slightly increased risk for non major bleedings compared to LMWH, and caution is advised when used in patients with gastrointestinal tract malignancies, especially upper gastro-intestinal tract malignancies. This review depicts actual considerations for anticoagulation in cancer patients to provide a rational for clinicians before treatment initiation.
\end{abstract}

Keywords DOAC · Rivaroxaban · Edoxaban · Apixaban

$\begin{array}{ll}\text { Abbreviations } \\ \text { AC } & \text { Anticoagulation } \\ \text { DOAC } & \text { Direct oral anticoagulants } \\ \text { LMWH } & \text { Low molecular weight heparin } \\ \text { PE } & \text { Pulmonary embolism }\end{array}$

\section{J. B. Jaeger $\cdot$ T. Jaeger}

Internal Medicine II-Oncology/Gastroenterology and Hepatology, Regional Hospital Feldkirch, Feldkirch, Austria

Ass. Prof. Priv. Doz. Dr. C. Feistritzer $(\bowtie)$

Internal Medicine V-Hematology and Oncology, Medical

University of Innsbruck, Anichstr. 35, 6020 Innsbruck

(Tyrol), Austria

clemens.feistritzer@i-med.ac.at
VKA Vitamin K antagonists

VTE Venous thromboembolism

Current antineoplastic therapy approaches have sustainably increased the survival rate by reducing tumour progression. Particularly venous thrombosis and subsequent pulmonary embolism (PE) are serious complications and the second cause of death after tumour progression in cancer patients and therefore require special attention.

After the diagnosis of an acute venous thromboembolism (VTE) starting anticoagulation treatment is immediately indicated to prevent thrombosis growth and embolism [1]. Vitamin K antagonists (VKA) are a substance class used as the first-line option for longterm anticoagulation due to their oral form, low price and broad experience in medical usage. However, VKA possess strong interaction potential with various food products as well as with numerous drugs, which make intensive coagulation checks and dosages adjustments necessary. Chemotherapy induced thrombocytopenia, emesis as well as direct drug-drug interactions of VKA with chemotherapy agents and antibiotics bear high risk of unintentional bleeding as well as recurrence of thrombosis.

In contrast, LMWH hardly interact with chemotherapy agents. Hence the results of the CLOT trial in 2003 showed the superiority of the anticoagulation with LMWH compared to VKA [2]. In this randomized comparison of the LMWH dalteparin showed significant decrease in VTE over a period of 6 months compared to VKA without a difference in major bleeding. Although the CLOT trial and other subsequent trials using LMWH in cancer patients indicate the usage of LMWH in cancer patients, many questions on anticoagulation in cancer patients still remain unanswered. Particularly questions on optimal duration of antico- 
agulation, the significance of incidental PE, concurrent thrombocytopenia or high risk for fatal bleeding are highlighted by Lee et al. and need to be investigated carefully in cancer patients regardless of the pharmacological substance prescribed [3]. Additionally, the treatment of incidental PEs and VTE is guided to be treated similar as patients with symptoms, except isolated subsegmental PEs. However, it has been proposed that patients with isolated subsegmental PEs may not need anticoagulation treatment [4].

An often-mentioned downside of LMWH is the fact that LMWH requires daily subcutaneous injection. Emerging from the assumption of discomfort for LMWH application Noble et al. have shown in a small study that the often presumed preference of oral drug application form over injection had only moderate importance $(13 \%)$. Interestingly most important was minimal interference with their chemotherapy (39\%) followed by low thrombosis recurrence (24\%) and low major bleeding risk (19\%) [5]. Therefore, the choice should be individualized respecting each patient's risk as well as preference.

With the invention of DOAC a new treatment option appeared. In the last decade DOAC have been developed and licenced already as the first-line option for anticoagulation in non-valvular atrial fibrillation and VTE. The striking advantage of DOAC is stable factor Xa inhibition (or IIa in dabigatran). However, initially DOACs were not specifically tested in patients with cancer-associated thrombosis and therefore the evidence to recommend DOAC in cancer patients remained uncertain. Already a retrospective subgroup meta-analysis of the rivaroxaban approval data by Larsen et al. [6] showed a potential benefit of DOAC treatment for VTE in patients with active cancer. However, the definition of active cancer in the initial studies was criticized as well as the comparison of the Xa antagonists with VKA instead of LMWH. Following the Hokusai-VTE Cancer trial in 2017, a non-inferiority trial of edoxaban vs dalteparin in patients suffering from active cancer and venous thromboembolism by Raskob et al. [7] showed superiority of DOAC over LMWH in patients with active cancer. Thromboembolism was less likely in the edoxaban group ( $n=41,7.9 \%)$ vs dalteparin group ( $n=59$,
$11.3 \%$; difference in risk -3.4 percentage points) but major bleeding occurred in 36 (6.9\%) vs 21 (4.0\%) patients. Subgroup analysis revealed that major bleedings in the edoxaban group occurred more often in patients with cancer of the upper gastrointestinal tract. In the Select-D Trial the efficacy of another DOAC, rivaroxaban vs. LMWH was tested in cancer patients [8]. Consistent with the results of the Hokusai-VTE Cancer trial, in the Select-D trial clinically relevant non-major bleedings were also increased in the rivaroxaban group; however, the risk of recurrence of thrombosis was reduced compared to treatment with LMWH. Similar results have been demonstrated for apixaban during the ASH meeting in San Diego 2018 [9]. Consequently, the use of oral Xa antagonists for the treatment of cancer-associated thrombosis can be recommended based on three published studies; however, caution is advised in patients with gastrointestinal tract malignancies, especially upper gastrointestinal tract malignancies because of the risk of bleeding (Fig. 1) [10].

\section{Choosing the right anticoagulation treatment}

Until now general guidelines on anticoagulation as of the Anticoagulation Forum favoured LMWH in all cancer patients at risk for VTE or history of VTE for at least a 6-month period [4]. The exact duration of anticoagulation in cancer associated VTE still remains uncertain and depends on various factors. Provoked VTE may be treated temporally, but spontaneous VTE in patients with active cancer may require permanent AC. Further investigations on the optimal duration for anticoagulation in cancer patients are still needed. Having DOACs as a treatment option for patients with active cancer, the advantages of oral ingestion as well as a reduced risk of VTE recurrence become striking.

Although DOACs usually do not need to be dose adjusted in renal failure dosage of LMWH and DOACs may be adapted and DOAC are contraindicated in creatinine clearance $<15 \mathrm{ml} / \mathrm{min}$ (edoxaban). Dose adjustment may be necessary with regard to drug-drug interactions (cytochrome P450 3A4 and P-glycoprotein interaction) such as fungistatics, antibiotics (macrolides, metronidazole) and certain chemotherapeutic agents [10]. Another reservation of DOAC

Table 1 Consideration on starting DOAC in cancer patients with VTE

Individual bleeding risk. No recent or high risk of bleeding (e.g. upper gastrointestinal cancer patients)

Initial treatment as in non-cancer patients (rivaroxaban $2 \times 15 \mathrm{mg}$ for 21 days, followed by $20 \mathrm{mg}$; edoxaban: LMWH for 5 days - switch to edoxaban $60 \mathrm{mg}$ )

Invasive procedures: There is no need for bridging to LMWH!

Hold anticoagulation in thrombocytopenic patients $<50.000 / \mu l$ (based on individual thrombosis risk). Check platelets on a regular basis and continue with anticoagulation on a regular basis if platelets $>50.000 / \mu \mathrm{l}$

For patients with a platelet count $<50.000 / \mu$ l half-therapeutic or prophylactic dose of LMWH is recommended

In patients with severe kidney failure creatinine clearance $<15 \mathrm{~mL} / \mathrm{min}$ DOACs are contra-indicated (as in noncancer patients)

Evaluate potential drug/drug interaction and prolonged vomiting based on the planned chemotherapy

Revaluate duration of $A C$ after 6 months and consider lifelong anticoagulation in cancer patients

Patient's preference with respect to dosage form 


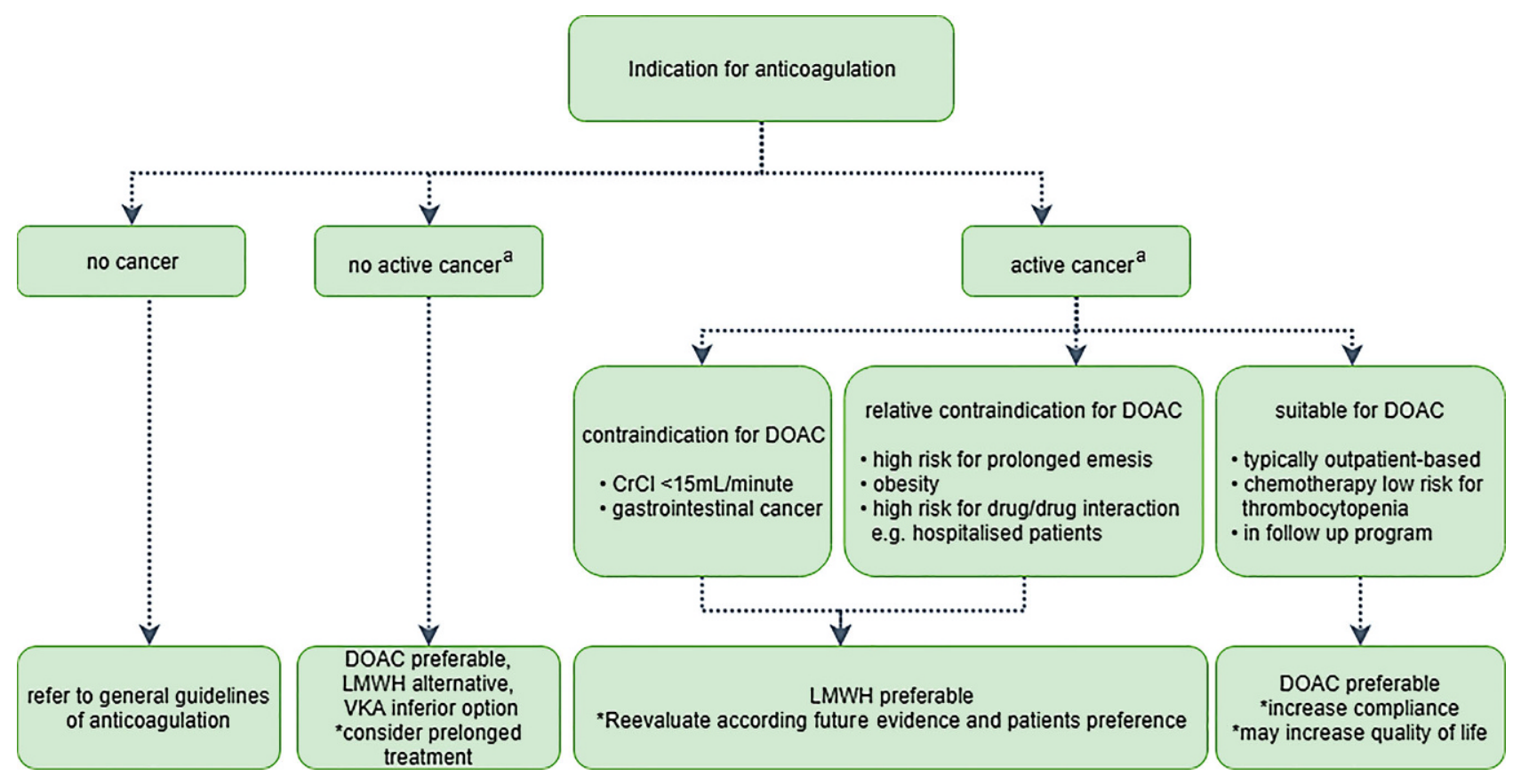

Fig. 1 Anticoagulation in cancer patients. 'Defined as cancer diagnosed within the previous 6 months; recurrent, regionally advanced, or metastatic cancer; cancer for which treat-

ment had been administered within 6 months, or hematologic cancer that was not in complete remission

treatment lies in its prime advantage of oral administration in the setting of chemotherapy induced prolonged vomiting. In this setting, LMWH might be still the preferred anticoagulation treatment option but a temporary switch from DOAC to LMWH would also be appropriate.

Furthermore, protamine works as a specific antidote for LMWH. In contrast, until now only idarucizumab is licensed as antidote for dabigatran [11]. The specific antidote andexanet alfa for reversal of Xa antagonists is not yet available (FDA approved, EMA approval ongoing) [12].

In patients with thrombocytopenia anticoagulation dose has to be adjusted individually on the risk of VTE. Full dose anticoagulation can be used up to a platelet count above $50 \mathrm{G} / \mathrm{l}$. The use of DOACs in cancer patients with severe thrombocytopenia is not recommended [13]. For patients with acute cancerassociated thrombosis and severe thrombocytopenia (platelet count $<50 \mathrm{G} / \mathrm{l}$ ) and a low risk of thrombus progression a reduction of the LMWH dose to $50 \%$ of the therapeutic dose or prophylactic dosing of LMWH is recommended. Furthermore, anticoagulation can be temporarily discontinued in patients while the platelet count is $<25 \mathrm{G} / 1$ (Table 1 ).

Funding Open access funding provided by University of Innsbruck and Medical University of Innsbruck.

Conflict of interest J.B. Jaeger, T. Jaeger and C. Feistritzer declare that they have no competing interests.
Open Access This article is distributed under the terms of the Creative Commons Attribution 4.0 International License (http://creativecommons.org/licenses/by/4.0/), which permits unrestricted use, distribution, and reproduction in any medium, provided you give appropriate credit to the original author(s) and the source, provide a link to the Creative Commons license, and indicate if changes were made.

Publisher's Note Springer Nature remains neutral with regard to jurisdictional claims in published maps and institutional affiliations.

\section{References}

1. Girard P, Decousus M, Laporte S, Buchmuller A, Hervé P, Lamer C, et al. Diagnosis of pulmonary embolism in patients with proximal deep vein thrombosis: Specificity of symptoms and perfusion defects at baseline and during anticoagulant therapy. Am J Respir Crit Care Med. 2001;164(6):1033-7.

2. Lee AYY, Levine MN, Baker RI, Bowden C, Kakkar AK, Prins $\mathrm{M}$, et al. Low-molecular-weight heparin versus a coumarin for the prevention of recurrent venous thromboembolism in patients with cancer. N Engl J Med. 2003;349(2):146-53. https://doi.org/10.1056/NEJMoa025313.

3. LeeAY, PetersonEA. Treatment of cancer-associated thrombosis. Blood. 2013;122(14):2310-7. http://www.ncbi.nlm. nih.gov/pubmed/23843493.

4. Khorana AA, Carrier M, Garcia DA, Lee AYY. Guidance for the prevention and treatment of cancer-associated venous thromboembolism. J Thromb Thrombolysis. 2016;41(1):81-91. http://www.ncbi.nlm.nih.gov/ pubmed/26780743.

5. Noble S, MatzdorffA, Maraveyas A, Pisa G, Pisa G. Assessing patients' anticoagulation preferences for the treatment of 
cancer-associated thrombosis using conjoint methodology. Haematologica. 2015;100(11):1486-92.

6. Larsen TB, Nielsen PB, Skjoth F, Rasmussen LH, Lip GYH. Non-vitamin $\mathrm{K}$ antagonist oral anticoagulants and the treatment of venous thromboembolism in cancer patients: A semi systematic review and meta-analysis of safety and efficacy outcomes. PLoS ONE. 2014;9(12):1-12.

7. Raskob GE, van Es N, Verhamme P, Carrier M, Di Nisio M, Garcia D, et al. Edoxaban for the treatment of cancerassociated venous thromboembolism. N Engl J Med. 2017; https://doi.org/10.1056/NEJMoa1711948.

8. Young AM, Marshall A, Thirlwall J, Chapman O, Lokare A, Hill C, et al. Comparison of an oral factor xa inhibitor with low molecular weight heparin in patients with cancer with venous thromboembolism: results of a randomized trial (SELECT-D). JClin Oncol. 2018;36(20):2017-23.

9. McBane RD, Wysokinski WE, Le-Rademacher J, Ashrani AA, Tafur AJ, Gundabolu K, et al. Apixaban, Dalteparin, in Active Cancer Associated Venous Thromboembolism, the ADAM VTE Trial. In: ASH Annual Meeting-Oral and poster abstracts-Session: 332 Antithrombotic Therapy: Management of Challenging Patients and Scenarios Hematology Disease Topics \& Pathways: Diseases, anticoagulant drugs, Bleeding and clotting, Therapies, Non-Biological, [Internet]. 2018. Available from: https://ash.confex.com/ash/ 2018/webprogram/Paper118808.html. Accessed December 2,2018
10. Short NJ, Connors JM. New oral anticoagulants and the cancer patient. Oncologist. 2014;19(1):82-93. https://doi. org/10.1634/theoncologist.2013-0239.

11. Pollack CV, Reilly PA, van Ryn J, Eikelboom JW, Glund S, Bernstein RA, et al. Idarucizumab for dabigatran reversal-full cohort analysis. N Engl J Med. 2017; https://doi. org/10.1056/NEJMoa1707278.

12. Steffel J, Verhamme P, Potpara TS, Albaladejo P, Antz M, Desteghe L, et al. The 2018 European Heart Rhythm Association PracticalGuide on the use of non-vitamin Kantagonist oral anticoagulants in patients with atrial fibrillation: executive summary. Europace. 2018;20(8):1231-42.

13. Samuelson BannowBT, LeeA, KhoranaAA,ZwickerJI, Noble $\mathrm{S}$, Ay C, etal. Management of cancer-associated thrombosis in patients with thrombocytopenia: guidance from the SSC of the ISTH. J Thromb Haemost. 2018;16(6):1246-9.

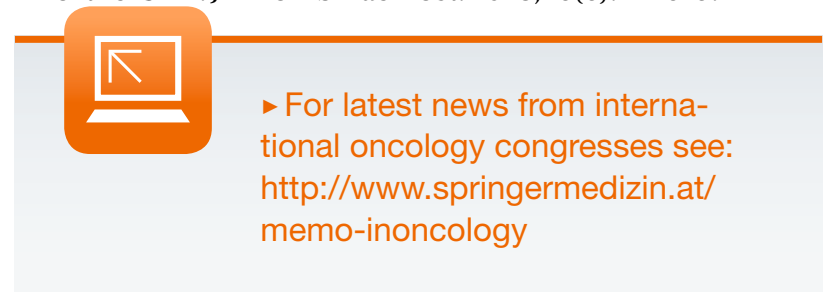

